\section{0}
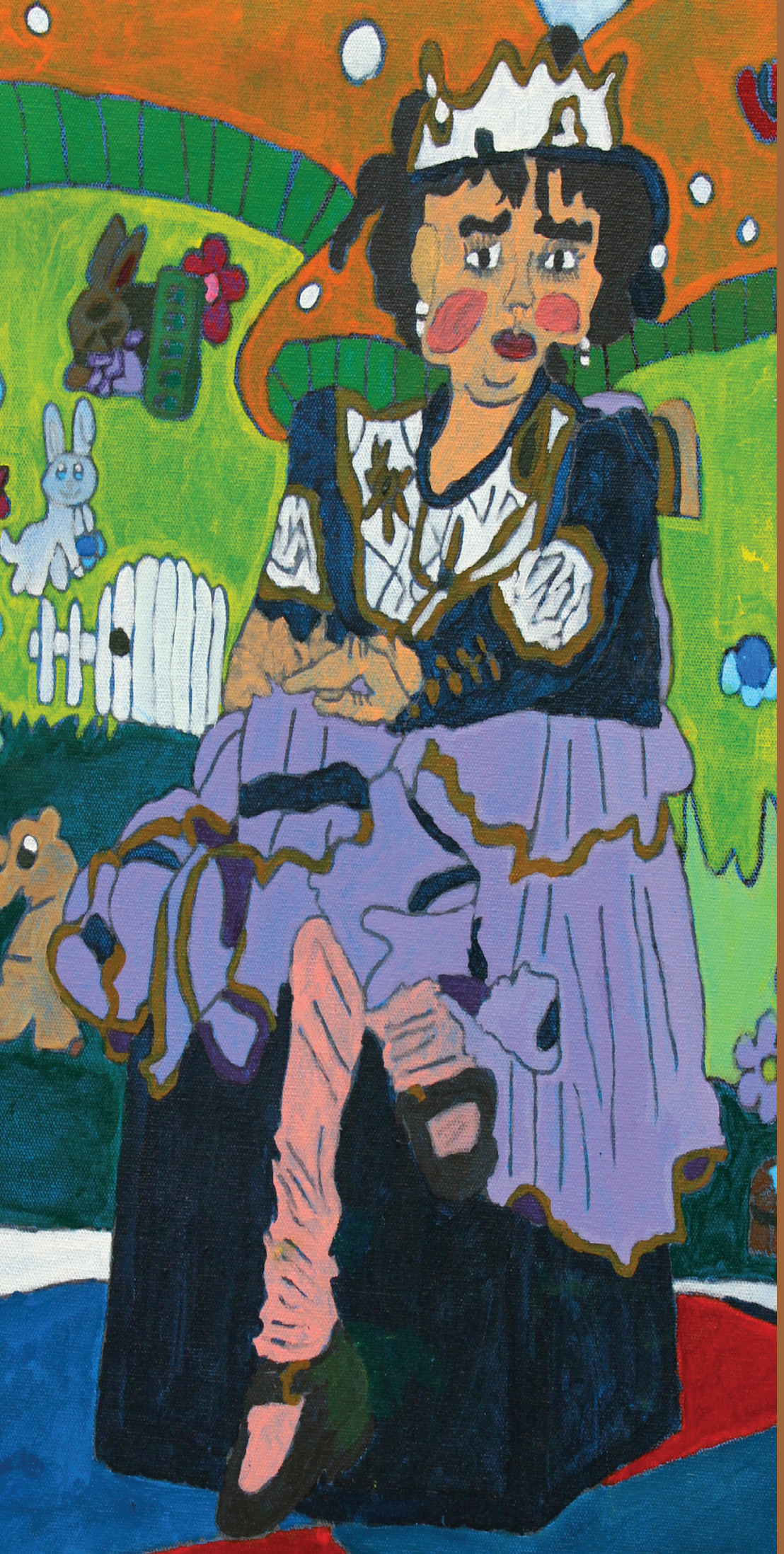

Leonor Córdoba

Psicóloga PH D.

Profesora Departamento de Estudios Interdisciplinarios,

Universidad del Tolima,

lcordobaa@ut.edu.co

\section{Patricia Vaca}

Psicóloga M. Sc. en Psicología Social Comunitaria Facultad de Psicología Universidad de La Sabana patriciavv@unisabana.edu.co.

\section{Richard Rosero}

Psicólogo. M. Sc. en Tecnología Educativa

Universidad de La Sabana

richardrosero@hotmail.com;

\section{Juana Gómez-Benito}

Psicóloga Ph D.

Profesora Departamento de Metodología de las Ciencias del Comportamiento, Facultad de Psicología e

Instituto de Investigación en Cerebro, Cognición y Conducta (IR.C), Universidad de Barcelona

juanagomez@ub.edu;

\section{María Clara Vargas}

Sociologa

Universidad Externado de Colombia

\section{Ramona Lucas-Carrasco}

Médico Psiquiatra. PhD en Psicología

Pare Sanitari Sant Joan de Déu

lucas.ramona@gmail.com

Clara Eugenia Bahamon

Arte sin Fronteras - Programa Talentos Especiales 


\title{
MUJERES CON DISCAPACIDAD: SIGNIFICADOS DE SU CONDICIÓN Y PRÁCTICAS DE INCLUSIÓN
}

\section{Women with disability: The meanings of their condition and social inclusion practices}

\author{
Fecha de recepción: 30 de julio de 2014 - Fecha de aprobación: 1 de diciembre de 2014
}

\begin{abstract}
RESUMEN
Existen más de 400 millones de mujeres alrededor del mundo con diferentes discapacidades, de las cuales el 80\% vive en países en desarrollo (UN, 2008). El objetivo de este estudio fue identificar los significados que han construido las mujeres con discapacidad en torno a su condición y las prácticas que realizan en relación con su inclusión y sus derechos. Se realizó un estudio mixto, en el que participaron 200 mujeres con discapacidad física que respondieron a dos cuestionarios, uno socio-demográfico y otro de calidad de vida. Se desarrollaron dos grupos focales y ocho entrevistas en profundidad. Los resultados muestran que la mayoría de participantes tienen un significado positivo de la discapacidad, la diferencian de la enfermedad, son independientes en sus actividades cotidianas, cuentan con escasa información sobre sus derechos, los mecanismos y escenarios para ejercerlos. Los resultados permiten formular recomendaciones para mejorar la inclusión social de mujeres con discapacidad.
\end{abstract}

PALABRAS CLAVE

Mujer, discapacidad, inclusión social, creencias, prácticas, derechos.

\section{ABSTRACT}

Worldwide there are over 400 million women with some form of disability, and of these, $80 \%$ live in developing countries (U.N, 2008). With its focus on social inclusion and rights the present study aimed to examine the meanings which disabled women ascribe to their condition and the activities they engage in. The procedure was based on mixed methods and the sample comprised 200 women with a physical disability who responded to two questionnaires, one socio-demographic and another concerning quality of life. Two focus groups were created and eight in-depth interviews were conducted. The results showed that most of the participants ascribed a positive meaning to their disability and did not regard it as an illness. They were independent as regards daily activities but had limited information about their rights, as well as about the mechanisms and social spheres through or in which such rights might be exercised. The findings enable recommendations to be made regarding how to improve the social inclusion of women with disability.

KEY WORDS

Woman, disability, social inclusion, beliefs, practices, rights. 
discriminación, puesto que ser mujer con discapacidad marca una

$\mathrm{P}$ or cuanto la dignidad humana es la norma básica de los Derechos Humanos, "el objetivo último desde la perspectiva de derechos es construir sociedades que sean auténticamente integradoras, sociedades que valoren la diferencia y respeten la dignidad y la igualdad de todos los seres humanos con independencia de sus diferencias" (Quinn \& Degener, 2002, p.12).

El derecho a la igualdad abarca diferentes áreas de las personas: su economía, interacción social, vida cotidiana y privacidad. Este derecho tiene un alcance distinto para hombres y mujeres, es decir, está subordinado al concepto de género. Por esta razón, es importante comprender la construcción social que se ha hecho del concepto género para entender la discriminación hacia las mujeres, así como las creencias, estereotipos y prácticas derivadas.

Al respecto, Arcos, Figueroa, Miranda y Ramos (2007) plantean que la teoría del género asume este concepto como una serie de categorías, hipótesis, interpretaciones y conocimientos construidos históricamente a partir de sexo. Igualmente, el género es entendido como una construcción simbólica que capta atributos biopsicosociales. Estos atributos influyen en la organización social y en las jerarquías de poder que marcan una serie de oportunidades, maneras de vivir y restricciones en los espacios sociales.

Dentro de los estudios acerca de género y discapacidad, las mujeres y niñas han sido, de alguna manera, ignoradas por los investigadores, lo que supone una violación al derecho de igualdad, pues promueve la discriminación. Es por ello que la investigación sobre discapacidad, desde el punto de vista de género, es un campo relativamente nuevo y con un interés creciente (Giménez \& Ramos, 2003; Soler, Teixeira \& Jaime, 2008). Hasta hace poco, las mujeres con discapacidad desde el punto de vista científico y político-social, no existían (López, 2008).

Las mujeres con discapacidad, experimentan una doble discriminación o vulnerabilidad (Ramiro, 2004), es decir, la discriminación de género sumada a la ocasionada por cuenta de la discapacidad, aspecto que origina barreras que obstaculizan el ejercicio de sus derechos y responsabilidades como ciudadanas, así como su participación en escenarios sociales (Soler et al., 2008; Confederación Española de Personas con Discapacidad Física y Orgánica -COCEMFE-, 2003). Ellas, según Ramiro (2004) "ocupan un estatus inferior en la sociedad, encontrándose en una situación de enorme desventaja social, económica, educativa y profesional" (p. 2), y además, al pertenecer a estos dos grupos, el de género y el de discapacidad, facilita "la construcción de roles, estereotipos y barreras de carácter psicosocial que frecuentemente limitan su plena integración a la vida social y el ejercicio de sus derechos" (Cruz, 2004, p. 154).

\section{En la misma dirección, Soler et al. (2008) plantean que:}

La realidad de las mujeres con discapacidad se ha definido por parte de los estudiosos de la materia por dos hechos (1) la invisibilidad, por cuanto la mujer con discapacidad ha permanecido, y permanece en gran medida 'invisible' para la sociedad y (2) la doble trayectoria de doble discriminación, como mujer y como persona, y añade barreras que dificultan el ejercicio de derechos y responsabilidades como personas, la plena participación social y la consecución de objetivos de vida considerados esenciales (p.1-2).

Asimismo, según COCEMFE, los gobiernos no han tenido en cuenta a las mujeres con discapacidad en las regulaciones contra la exclusión y la discriminación, y tampoco se han generado planes de igualdad en ámbitos como la educación, el trabajo, la salud y la asistencia social. Lo anterior parece indicar que las legislaciones de los Estados también han pasado por alto el binomio mujer y discapacidad y, por esta razón, se hace importante fomentar mecanismos jurídicos y legales en pro de su inclusión en la sociedad, enmarcado en los derechos de las personas con discapacidad (ONU, 1993; ONU, 2006) y de los derechos de las mujeres previstos en la convención sobre la eliminación de todas las formas de discriminación contra la mujer-CEDAW-(ONU, 1979), en los que se enfatiza en las obligaciones de los Estados Parte en relación con la educación (artículo 10), el empleo (artículo 11), la atención de la salud (artículo 12) y la eliminación de la discriminación contra la mujer en todas las cuestiones referidas con el matrimonio y las relaciones familiares (artículo 16). Normatividad que se aplica obviamente, a las mujeres con discapacidad (Quinn \& Degener, 2002).

Con miras a hacer valer los derechos de las mujeres con discapacidad, surgen de la CEDAW, dos Recomendaciones fundamentales: la $\mathrm{n}^{\circ} 18$ que convoca a los Estados Parte a informar sobre las medidas adoptadas para hacer frente a la situación particular de las mujeres con discapacidad y asegurar que puedan participar en todos los aspectos de la vida social y cultural; y la $\mathrm{n}^{\circ} 24$ en la que se enfatiza que "debe prestarse especial atención a las necesidades en materia de salud de las mujeres con discapacidad física o mental" (Quinn \& Degener, 2002, p.120).

En este orden de ideas, la discapacidad "es una construcción social que limita e impide que las personas con discapacidad se incluyan, decidan o diseñen con autonomía su propio plan de vida en igualdad de oportunidades" (Victoria, 2013, p. 1093). Desde este punto de vista, se promueve concebir a la persona con discapacidad como sujeto de derechos y no como un problema. Asumir esta perspectiva de comprensión de los derechos humanos, con el propósito de facilitar los procesos de inclusión social de mujeres con discapacidad, implica, entre otras tareas, identificar y analizar las creencias y prácticas inmersas en dichos procesos, razón por la que se hace necesario, indagar en torno a la experiencia personal de las mujeres con discapacidad, sus vidas y sus vivencias sociales, mediante un abordaje que permita tener en cuenta situaciones únicas e individuales enmarcadas al interior de un contexto social y cultural (López, 2008).

Con este marco de referencia, el objetivo de la presente investigación fue examinar tanto la construcción de significados como las prácticas empleadas por mujeres colombianas con discapacidad física y sus familias, en torno al reconocimiento y el ejercicio de sus derechos y responsabilidades en los diferentes contextos en los que se encuentran inmersas: artes, cultura, deporte, educación, salud, tiempo libre y trabajo. 
2011; Power, Green, the WHOQOL-DisGroup, 2010a; Power, Green,

Tipo de estudio

El presente estudio se inscribe en los métodos mixtos, que permiten integrar los hallazgos obtenidos desde dos aproximaciones metodológicas diferentes, una de tipo cuantitativo, y otra, de orden cualitativo. Según Teddlie y Tashakkori (2006), "la principal ventaja de los métodos mixtos de investigación radica en que son capaces de responder simultáneamente a preguntas confirmatorias y exploratorias, y por consiguiente, verificar y generar teoría en el mismo estudio" (p. 20).

\section{Participantes}

La selección de la muestra se llevó a cabo a través de organizaciones e instituciones para personas con discapacidad, en cuatro ciudades capitales de Colombia: Bogotá (27.4\%), Medellín (35.3\%), Cali (21.9\%) y Barranquilla (14.4\%). Los criterios de inclusión fueron que la mujer estuviera en una edad productiva (18-62 años) y que presentara una discapacidad física (movilidad reducida, discapacidad auditiva o visual). El periodo de inclusión fue desde marzo de 2009 hasta octubre de 2009. En la fase cualitativa participaron 24 mujeres, y en la cuantitativa 200 mujeres. El muestreo fue de tipo no probabilístico, sobre la base de la disponibilidad de participación de las mujeres. El tamaño de la muestra en la fase cuantitativa fue de 200 mujeres.

\section{Instrumentos}

Se diseñaron y aplicaron dos protocolos, uno para la entrevista en profundidad, y otro, para los grupos focales, a partir de las tres categorias de análisis previas: Significados acerca de ser mujer en condición de discapacidad, inclusión y derechos, y creencias en torno a los roles de la mujer. También, se empleó un Cuestionario Sociodemográfico, que constaba de dos partes, la primera, anexa al Cuestionario WHOQOL, que indagaba sobre aspectos como: perfil sociodemográfico, situación en la que vive y apoyos que recibe la mujer; y la segunda, incluía unos reactivos suplementarios construidos por los investigadores para indagar sobre la conformación familiar y los espacios de participación de las mujeres; y el Cuestionario World Health Organization Quality of Life (WHOQOL).

El WHOQOL es un cuestionario de calidad de vida, genérico y multidimensional, creado a principios de los años 90 por la División de Salud Mental de la Organización Mundial de la Salud y 15 países a escala internacional (WHOQOL Group, 1995,1998), siendo España uno de ellos (Lucas-Carrasco, 1998). Existe una versión corta del WHOQOL que se empleó en este estudio: el WHOQOL-BREF, que contiene 24 preguntas, agrupadas en cuatro áreas: 1. Física, 2. Psicológica, 3. Relaciones Sociales y 4. Ambiente, y dos preguntas generales, una sobre calidad de vida global y otra sobre satisfacción con el estado de salud (Skevington, Lotfy, O'Connell; WHOQOL-Group, 2004). En la actualidad, este instrumento existe en más de 40 idiomas diferentes y se han desarrollado módulos específicos para personas mayores (Lucas-Carrasco, 2007) y recientemente, para personas con discapacidad (Lucas-Carrasco, Eser, Hao, McPherson, Green, \& Kullmann, the WHOQOL-DisGroup, 2010b). La metodología utilizada para su desarrollo permite realizar comparaciones cross-culturales válidas (Power, Quinn, Schmidt \& the WHOQOL-OLD Group, 2005; Power, Bullinger, Harper \& The WHOQOL Group, 1999).

\section{Procedimiento}

El estudio se desarrolló en dos fases. La primera, cualitativa, en la que participaron 24 mujeres; de ellas, 16 conformaron dos grupos focales, cada uno con 8 mujeres, durante una sesión, con una duración de dos horas y 15 minutos. Las 8 mujeres restantes fueron entrevistadas a profundidad por profesionales entrenados. Cada sesión fue grabada, con la autorización de los entrevistados, mediante el consentimiento informado, el cual fue redactado de acuerdo con las normas éticas vigentes en Colombia en el Código de Ética del Psicólogo (Ley 1090 de 2006). A partir de la primera fase, se crearon ítems que completaron el cuestionario socio-demográfico. La segunda fase, correspondió a la hetero-aplicación a las participantes de los cuestionarios Socio-demográfico y de Calidad de Vida.

\section{Análisis de los datos}

Los resultados fueron analizados en tres momentos.

1. Análisis de la información obtenida en las entrevistas en profundidad y en los grupos focales. Este análisis se realizó desde los planteamientos de la teoría fundamentada (Glasser \& Strauss, 1967). Inicialmente se realizó un microanálisis de la narrativa, que permitió la realización de la codificación abierta, desde la cual se analizaron las categorías deductivas y surgieron las categorías inductivas. Finalmente, la codificación axial y selectiva posibilitó establecer las relaciones entre las categorías.

2. Análisis de la información obtenida en la hetero-aplicación a las participantes de los cuestionarios Socio-demográfico y de Calidad de Vida a partir de la realización de análisis descriptivo de las variables.

3. Análisis mixto de los resultados cuantitativos y cualitativos a partir de la estrategia de complementación propuesta por Bericat (1998).

\section{RESULTADOS}

Los resultados se describen en dos apartados. El primero, corresponde a la caracterización socio-demográfica de las mujeres con discapacidad que participaron en el estudio; el segundo, da cuenta de los principales hallazgos en torno a las categorías de análisis previamente definidas por los investigadores: significados de la discapacidad; e inclusión y derechos. Además, se integran los resultados obtenidos a través del Cuestionario de Calidad de Vida (WHO, 1998), con los de Grupos Focales y las Entrevistas en Profundidad, en función del diseño mixto desde donde se planteó el presente estudio. 
Participaron 200 mujeres con discapacidad, residentes en cuatro ciudades capitales de Colombia (Bogotá, Medellín, Cali y Barranquilla), de las cuales el $36.5 \%$ presentaba una limitación física, $33.0 \%$ limitación visual y $30.5 \%$ limitación auditiva. En la Tabla 1 aparecen las características socio-demográficas de las mujeres.

Tabla 1. Características socio-demográficas de las participantes ( $N=200)$.

\begin{tabular}{|c|c|}
\hline \multicolumn{2}{|c|}{ Edad } \\
\hline Media & 33.87 (D.S.12.167) \\
\hline Rango & 18-62 años \\
\hline \multicolumn{2}{|c|}{ Estatus socioeconómico (n, \%) } \\
\hline Bajo & $106(53.0)$ \\
\hline Medio & $82(41.0)$ \\
\hline Alto & $12(6.0)$ \\
\hline \multicolumn{2}{|c|}{ Nivel educativo (n, \%) } \\
\hline Ninguno & $2(1.0)$ \\
\hline Educación especial & $2(1.0)$ \\
\hline Primaria & $25(12.5)$ \\
\hline Secundaria & $90(45.0)$ \\
\hline Estudios técnicos/tecnológicos & $56(28.0)$ \\
\hline Universitarios & $15(7.5)$ \\
\hline Postgrado & $10(5)$ \\
\hline \multicolumn{2}{|c|}{ Estado civil (n, \%) } \\
\hline Soltera & $140(70.0)$ \\
\hline Casada & $23(11.5)$ \\
\hline Unión Libre & $17(8.5)$ \\
\hline Separada & $17(8.5)$ \\
\hline Divorciada & $1(0,5)$ \\
\hline Viuda & $2(1,0)$ \\
\hline \multicolumn{2}{|c|}{ Tipo de discapacidad (n, \%) } \\
\hline Auditiva & $61(30.5)$ \\
\hline Visual & $66(33.0)$ \\
\hline Física & $73(36.5)$ \\
\hline
\end{tabular}

La tabla 2, da cuenta de las condiciones en las que viven las participantes y los apoyos que ellas requieren.

Significados de la discapacidad

Las participantes comentaron que la discapacidad, no está en las piernas o en la falta de visión o audición (en la limitación), sino en la mente de las personas o en la mirada de la sociedad "la limitación puede ser física... pero si yo me propongo a hacer algo, lo hago"(EP:Mo6)'; "la sociedad es la que ve la limitación en la persona discapacitada" (GF1:M11) ${ }^{2}$, aspecto que guarda una relación importante con un paradigma nuevo en la concepción de la discapacidad,

1. EP: significa entrevista en profundidad y $\mathrm{M}$ : corresponde a mujer con un número asignado a cada participante, para facilitar la identificación del relato, preservando el anonimato. Esto de aquí en lo sucesivo. que trasciende el modelo biomédico tradicional, en que la discapacidad se focalizaba en la persona, en su limitación o deficiencia. Contrariamente, el paradigma nuevo centra su atención en la dimensión social, en las relaciones y en los apoyos, logrando ir más allá de un simple problema individual. Este paradigma no se centra en las especificidades y particularidades de cada tipo de discapacidad, sino que parte de los contextos en donde dicho fenómeno se da como consecuencia de la "comparación con la norma social (el cuerpo ideal, sano y capaz)” (IMSERSO, 2002, p.51).

Tabla 2. Condiciones en las que viven las participantes y apoyos requeridos.

\begin{tabular}{l|}
\hline \\
\hline En casa - sin apoyos \\
\hline En casa -con apoyos informales \\
\hline En casa -con apoyos formales \\
\hline Institución especializada \\
\hline Residencia \\
\hline Otro \\
\hline Total
\end{tabular}

\begin{tabular}{|c|}
\hline Frecuencia \\
\hline 115 \\
\hline 75 \\
\hline 5 \\
\hline 1 \\
\hline 2 \\
\hline 2 \\
\hline 200 \\
\hline
\end{tabular}

Porcenteje

\begin{tabular}{|c|}
\hline 57,5 \\
\hline 37,5 \\
\hline 2,5 \\
\hline, 5 \\
\hline 1,0 \\
\hline 1,0 \\
\hline $\mathbf{1 0 0 , 0}$ \\
\hline
\end{tabular}

En concordancia con esta concepción de discapacidad, se observa que el $65.7 \%$ de las mujeres participantes se percibe en condición de discapacidad, pero no considera que esté enferma (tabla 3), es decir, la mayoría de las participantes, diferencia la discapacidad de la enfermedad. Coincidiendo con algunos de sus relatos, en los que enfatizan que discapacidad es diferente a enfermedad "lo que pasa es que tenemos una discapacidad pero no somos anormales" (GF2:M18).

Conviene resaltar que el $8.6 \%$ de la muestra percibe que no se encuentra en condición de discapacidad, ni está enferma. Aspecto que puede relacionarse con una representación de la discapacidad que coincide con el paradigma nuevo en el cual la discapacidad está en la interacción contexto-individuo, es decir, que para estas mujeres existen barreras en el entorno que limitan su actividad y restringen su participación. Este dato también da cuenta de la pluralidad en la comprensión y la vivencia de la discapacidad.

Tabla 3. Distribución de la muestra en función de la relación entre la percepción que tienen las participantes de su condición de discapacidad y su salud.

\begin{tabular}{|c|c|c|c|c|c|}
\hline $\begin{array}{l}\text { Percepción del } \\
\text { estado de salud }\end{array}$ & \multicolumn{4}{|c|}{ Percepción subjetiva de discapacidad } & \multirow[t]{2}{*}{ Total } \\
\hline & & & $\mathrm{Si}$ & No & \\
\hline Enferma & $\mathrm{N}(\%)$ & & $(23,2)$ & $5(2,5)$ & $51(25,8)$ \\
\hline Sana & $\mathrm{N}(\%)$ & 130 & $(65,7 \%)$ & $17(8,6 \%)$ & $147(74,2 \%)$ \\
\hline Total & $\mathrm{N}(\%)$ & 176 & $(88,9 \%)$ & $22(11,1 \%)$ & $198(100,0 \%)$ \\
\hline
\end{tabular}

La percepción de la condición de salud con la percepción de la condición de discapacidad no se encuentran relacionadas al nivel de significación del $5 \%\left(\chi^{2}=0,119, p\right.$-valor $\left.=0,739\right)$.

2. GF: significa grupo focal y M: corresponde a mujer con un número asignado a cada participante, para facilitar la identificación del relato, preservando el anonimato. Esto de aquí en lo sucesivo. 
En algunos casos, al preguntarles a las mujeres acerca de qué tan visible consideraban que era su discapacidad, respondieron que esto dependía del contexto; por ejemplo, daban cuenta de que la familia no percibía la discapacidad, cuando ésta era congénita, o en función del tiempo que habían compartido con la mujer con discapacidad. En otro tipo de contexto, como el laboral o el educativo, la discapacidad se hace más visible e invisibiliza a la vez a la persona; la discapacidad se convierte en una categoría social que invade la representación de un ser humano integral. Las diferencias en relación con el contexto pueden explicar que más de la mitad de las participantes (54.4\%) informó que su discapacidad era moderada o poco visible tal como se observa en la tabla 4. Adicionalmente, se evidenció que la percepción de las mujeres sobre la visibilidad de su discapacidad muchas veces está determinada por las ayudas técnicas que ellas utilizan, tales como lentes, bastones, prótesis, entre otros, que sin lugar a dudas, son indispensables para su desenvolvimiento en la vida cotidiana y favorecen su inclusión social, pero, inevitablemente, hacen más notoria la limitación ante los ojos de los demás.

Tabla 4. Percepción de las mujeres sobre la visibilidad y el efecto de la discapacidad en sus vidas.

\begin{tabular}{|l|c|c|}
\hline \multicolumn{1}{|c|}{ Visibilidad } & \multicolumn{2}{c|}{ Visibilidad } \\
\hline Nada & 17 & 9,2 \\
\hline Un poco & 48 & 26,1 \\
\hline Moderadamente & 52 & 28,3 \\
\hline Bastante & 37 & 20,1 \\
\hline Totalmente & 30 & 16,3 \\
\hline Total & 184 & 100,0 \\
\hline & Efecto & \\
\hline Casi nada & 38 & 20,5 \\
\hline Levemente & 41 & 22,2 \\
\hline Moderadamente & 63 & 34,1 \\
\hline Severamente & 23 & 12,4 \\
\hline Profundamente & 20 & 10,8 \\
\hline Total & 185 & 100,0 \\
\hline
\end{tabular}

Con relación al efecto que tiene la discapacidad en la vida de las mujeres (Tabla 4), el 34,1\% de ellas reportó que la condición de discapacidad afecta moderadamente su vida; contrariamente, un 10,8\% de las participantes informó que la discapacidad afectaba profundamente su vida. Los relatos de las mujeres dan cuenta del peso que tienen las diferencias individuales tanto personales como contextuales a la hora de valorar el efecto que tiene en sus vidas la discapacidad "como tal la discapacidad se sufre y se lleva como uno la quiera vivir" (GF1:M18).

A partir de la premisa de que la Calidad de Vida es un constructo multidimensional que incluye entre otras dimensiones bienestar emocional, desarrollo personal, autodeterminación, inclusión social y derechos, se complementó la información obtenida a partir de los grupos focales y la entrevista en profundidad con las respuestas de las participantes frente a los indicadores de calidad de vida contenidos en el Cuestionario de Calidad de la Vida de la OMS (1998). Las participantes, en general, reportaron sentirse satisfechas con su bienestar emocional, reflejado entre otros aspectos en pensamientos positivos, sentido de vida y vitalidad, con porcentajes de $62.3 \%, 67.7 \%$ y $65.6 \%$, respectivamente. $\mathrm{Al}$ respecto, en los relatos de las mujeres se evidencia como alrededor de los significados que ellas construyen frente a la discapacidad, coexisten sentidos de pérdidas y de ganancias, que finalmente, se traducen en el sentido que le dan a su vida.

Con relación a los indicadores de calidad de vida que tienen que ver con el desarrollo personal y la autodeterminación, se encontró que el mayor porcentaje de las participantes (70.0\%), se consideran autónomas y en consonancia con esto, toman sus propias decisiones de vida $(74.5 \%)$. A lo largo del estudio, las mujeres insistieron en la importancia de su autonomía "en mi casa yo soy muy activa...yo no dejo que todo me lo hagan" (EP: M5); como favorecedora de su realización personal, y de su inclusión en la sociedad.

Inclusión social y derechos

Teniendo como marco de referencia la concepción, de que la inclusión social es real en la medida en que todas las personas puedan ejercer sus derechos y cumplir sus responsabilidades, se indagó con las mujeres sobre su ejercicio de los principales derechos: trabajo, educación, salud, accesibilidad, recreación, cultura y tiempo libre.

En la tabla 5 se observa que la ocupación principal de las mujeres participantes es la de estudiante (29,6\%), seguido de un $23.8 \%$ de la muestra que cuenta con un trabajo remunerado, mientras que 1.6\% de las participantes no tiene ocupación. El 19\% de las mujeres estaba desempleada. Durante la indagación cualitativa, las mujeres enfatizaron en la exclusión, por cuenta de la carencia de una política pública orientada a favorecer la vinculación laboral de personas con discapacidad "Yo digo que más que todo es la sociedad la que no acepta a la gente discapacitada, no nos da oportunidades de vida, ni de empleo" (GF1:M8). Otras mujeres narraron en las entrevistas, que al no contar con oportunidades de un empleo remunerado coherentemente con sus capacidades, deben "conformarse" con asumir oficios secundarios que no corresponden para nada con su cualificación, y que en buena medida, corresponden a tareas mecánicas y repetitivas, sin posibilidades de ascenso o de cambio, o en otros casos, se ven obligadas a vincularse a tareas domésticas o al mercado laboral informal, predominante en Colombia.

Un buen número de participantes manifestó inconformidad en cuanto a las numerosas y frecuentes oportunidades de capacitación, pero, sin posibilidades de poder aplicar los conocimientos adquiridos en un empleo remunerado o en una iniciativa de autogestión laboral: "...pero de qué sirve tener tantos cursos si uno no los puede ejercer laboralmente..." (EP: M11). En general, las mujeres participantes se sienten satisfechas con su capacidad de trabajo (56.8\%), sin embargo, ellas informan que la sociedad no les brinda oportunidades para demostrar esa capacidad. 
Tabla 5. Distribución de la muestra en función de la ocupación principal de las mujeres y su participación en diversos escenarios sociales.

\begin{tabular}{l|c|c|}
\hline \multicolumn{1}{c|}{ Ocupación } & Frecuencia & Porcentaje \\
\hline Trabajo remunerado & 45 & 23,8 \\
\hline Trabajo no remunerado & 5 & 2,6 \\
\hline Desempleada & 36 & 19,0 \\
\hline Estudiante & 56 & 29,6 \\
\hline Ama de casa & 24 & 12,7 \\
\hline Pensionada & 11 & 5,8 \\
\hline Ninguna & 3 & 1,6 \\
\hline Otra & 9 & 4,8 \\
\hline Total & 189 & 100,0 \\
\hline & Espacios de participación \\
\hline Deporte & 24 & 12,0 \\
\hline Teatro & 1 &, 5 \\
\hline Danza & 8 & 4,0 \\
\hline Música & 8 & 4,0 \\
\hline Artes plásticas & 6 & 3,0 \\
\hline Manualidades & 7 & 3,5 \\
\hline Grupos religiosos & 19 & 9,5 \\
\hline Grupos políticos & 3 & 1,5 \\
\hline Más de un escenario & 111 & 55,5 \\
\hline Ninguno & 13 & 6,5 \\
\hline Total & 200 & 100,0 \\
\hline & & \\
\hline
\end{tabular}

Otras mujeres informaron sobre experiencias de trabajo, en las que no se ha considerado la eliminación de ciertas barreras que limitan su actividad y restringen su participación. Por ejemplo, una de las participantes mencionó que las jornadas de trabajo eran demasiado extensas y le demandaban estar en una sola posición (sentada o de pie) durante gran parte del tiempo, aspecto que iba en detrimento de su salud y su condición; otra participante señaló la falta de adecuación de su sitio de trabajo.

Contrariamente, otras mujeres informaron que su condición no se constituye en obstáculo para que las organizaciones valoren su trabajo: "en la empresa donde estoy trabajando ellos me tratan como la persona que soy, no como discapacitada" (GF2: M9); soy modelo lingüístico para personas sordas... llevo ya 13 años en este empleo" (GF2: M24).

Con relación al derecho a la educación, si bien un $45 \%$ de las mujeres participantes terminó sus estudios de secundaria, y el $28 \%$, culminó el nivel técnico o tecnológico, solo el 7.5\% de las mujeres (15 de 200) finalizaron sus estudios universitarios y el $5 \%$ sus estudios de postgrado (10 de 200). Esto significa que las oportunidades de acceso a la educación superior de las mujeres con discapacidad, son mínimas o existen barreras en el entorno que las limitan aún más. $\mathrm{Al}$ respecto, una de las participantes informó: "cuando quise entrar a la Universidad me dijeron que las personas sordas no podían entrar a la universidad" (GF2:M24).
En cuanto a la participación de las mujeres en escenarios de tiempo libre en general, y grupos religiosos o políticos, se encontró que el mayor porcentaje (55.5\%), participa en más de un escenario (p. ej. Deporte y música, deporte y danza, deporte y manualidades, entre otros), seguido del $12 \%$ que práctica algún deporte. En el otro extremo, los escenarios de menor participación son teatro (0.5\%) y grupos políticos (1.5\%).

El mayor porcentaje de las participantes (31\%) manifestó que no está ni satisfecho ni insatisfecho con los servicios de salud, información que se relaciona con algunos relatos de las mujeres durante la indagación cualitativa que coinciden al afirmar que no existe en el servicio de salud en el país, profesionales especializados en la atención a la discapacidad, ni tampoco el sistema de salud proporciona a los usuarios con discapacidad el derecho a tener un tratamiento continuo que facilite el seguimiento de su condición: “....lo pasean a uno de médico en médico" (EP:M2).

\section{DISCUSIÓN}

El binomio mujer - discapacidad, debe leerse a la luz del nuevo paradigma de la discapacidad (Schalock, 2003) y del modelo social que lo enmarca (Verdugo, 2003). Este modelo rechaza la reducción de la discapacidad al déficit individual como lo proponía el modelo médico tradicional, y conceptualiza la discapacidad como una construcción a través de las actitudes sociales, las políticas y prácticas (Hann, 1985, 1993, 1996; Morris, 1991; Oliver, 1996 citados por Feldman y Tejar, 2003). Esto significa que una mujer es "discapacitada" no por su condición de salud, sino por las barreras que la sociedad le impone, limitando su actividad y restringiendo su participación en los diversos contextos en los que interactúa (OMS-CIF, 2001). Una mujer es "discapacitada" en la medida en que la sociedad no respeta sus derechos: a contar con un empleo digno, a acceder a la educación en los diversos ámbitos y niveles, a participar en la vida social y cultural de su comunidad, a acceder a los servicios de salud, entre otros.

Por esta razón, esta investigación reafirma la importancia de asumir el estudio del eje temático mujer-discapacidad desde los Derechos Humanos y simultáneamente desde la perspectiva de género, por cuanto incorporar esta perspectiva posibilita la concreción de políticas, planes, proyectos, programas y acciones que respondan efectivamente a la condición de mujer, teniendo como marco de referencia los principios universales de dignidad e igualdad, que en buena medida a lo largo de la historia, se han desconocido por parte de profesionales, investigadores, el Estado y la sociedad en general, dando lugar con algunos matices, a la condición de doble vulnerabilidad o doble discriminación de las mujeres con discapacidad.

Esto es apoyado por la conclusión a la que llega Cisternas (2013) en su análisis sobre la salud global, el género y los derechos humanos: "un cambio profundo debe gestarse en el sistema social, convencido que la equidad de género en el ámbito de la salud y otros derechos relacionados es fundamental para una sana convivencia en la comunidad" (p.153)

3. El entrecomillado es de los autores, para llamar la atención sobre la diferencia entre mujeres discapacitadas y mujeres con discapacidad. 
Los resultados de la presente investigación se presentan bajo la premisa que la discapacidad es una "realidad social y personal, plural, diversa y distinta" (Samaniego, 2006, p.106), y que no acepta generalizaciones.

Las mujeres responden a su condición de discapacidad de diferentes maneras: algunas la asumen como un reto y demuestran que son capaces, mientras que otras la experimentan como una condición de vulnerabilidad que preferirían esconder o mantener en privado (Feldman \& Tegart, 2003; Williams \& Upadhyay, 2003 citados por Banks, 2003), y para otras, la discapacidad es una parte vital de su identidad (Williams \& Upadhyay, 2003). En concordancia con esto, se encontró que las mujeres participantes otorgan diferentes significados a la discapacidad y su vivencia, y esto parece estar mediado por factores como su esperanza de vida, además de categorías de análisis sociodemográfico como tipo de discapacidad, lugar de residencia, conformación familiar, todas estas, condiciones de importancia, que canalizan cualquier interpretación desde la diversidad humana.

Con relación al tipo de discapacidad, la narrativa de las participantes hizo explícita una mayor apropiación de apoyos del entorno por parte de las mujeres con discapacidad visual, independientemente de la ciudad donde residían. Ellas dieron cuenta, asimismo, de mayores espacios de integración, que podrían casi calificarse de "agremiación", en la búsqueda de mejores condiciones de calidad de vida, pero sin que estos espacios se convirtieran en guetos o comunidades cerradas. En dirección contraria, la literatura científica internacional reporta que existen grupos de mujeres con discapacidad auditiva, denominada Comunidad de Sordas, que se autoperciben con un estatus minoritario (Corbett, 2003 citado por Banks, 2003).

Igualmente, se resaltan los hallazgos referidos a la auto-percepción de las mujeres sobre la visibilidad de su discapacidad. El 54.4\% de las participantes reportaron que su discapacidad era poco o moderadamente visible, pero lo que es más importante aún, es que relativizaron este efecto en el marco de su propia ecología. Similarmente, un 76,8\% de las mujeres informaron que el efecto de la discapacidad sobre sus vidas era casi nulo, leve o moderado. Esto sumado a los pensamientos positivos y el sentido de vida, del que dan cuenta los relatos y las respuestas de estas mujeres, se constituyen en estrategias resilientes que deben ser capitalizadas para favorecer cambios en políticas y programas orientados a la inclusión social de este colectivo.

Estas auto-percepciones están apoyadas por los hallazgos de Gordon, Feldman \& Crose (1998) con relación a la percepción que un grupo de 40 mujeres con discapacidad, entre 28 y 79 años de edad, tenían de sí mismas y los significados en torno a la discapacidad. Los investigadores identificaron que en el momento de ser diagnosticadas, las mujeres cambian sus apreciaciones acerca de la discapacidad, además existían cambios en la manera de ver y asumir su estilo de vida, y sufrían transformaciones emocionales individuales que implicaban la redefinición del concepto que tenían de ellas mismas.

Conviene para futuros estudios, o en una segunda fase de la presente investigación, profundizar en aspectos como la edad en la que fueron "diagnosticadas" estas mujeres, cómo se les dio el diagnóstico y la manera en que tanto ellas como sus familias lo asumieron, pues, al parecer, estas variables marcan una diferencia en la significación de la discapacidad, y en las prácticas en torno a su inclusión desde la perspectiva de derechos. Esta indagación, acompañada de la evolución que ha tenido el término discapacidad a lo largo de sus vidas y a su percepción de la misma, aportaría suficientes elementos de juicio para comprender su realidad actual y sus estrategias resilientes en un mundo excluyente.

Es importante mencionar la importancia del acceso a la educación y a la cualificación profesional u ocupacional de las mujeres con discapacidad con miras al acceso a un empleo digno (ONU, 2006; ONU, 1979), que supere la antinomia discapacidad - no discapacidad. Esto es un aspecto de especial interés en cuanto a las prácticas y empoderamiento de las mujeres con discapacidad en torno a sus derechos y lograr la igualdad de oportunidades.

En este estudio, un buen número de las participantes coinciden en afirmar que estas oportunidades son apropiadas siempre y cuando se ofrezcan en consonancia con sus necesidades reales, y adicionalmente, es indispensable que respondan a las necesidades del mercado de trabajo, con el fin último de contribuir a su inclusión laboral y su plena vinculación a una vida económicamente activa y autónoma, tanto en el presente como en el futuro. Conviene destacar aquí que sólo el 7.5\% de las participantes ha logrado concluir sus estudios universitarios, y sólo el $5 \%$ ha finalizado su postgrado. En buena medida esta situación responde a las dificultades que atraviesan las mujeres, por ejemplo, con limitación visual, para presentar los exámenes de ingreso a la educación superior. Además, son muy limitadas las experiencias de educación inclusiva en las universidades de Colombia.

En lo que tiene que ver con la salud, las participantes demandaron en primer lugar, una mayor apropiación de la condición de discapacidad, por parte de los servicios de atención en salud y de los profesionales, en el marco obviamente de la diversidad. Las mujeres creen que no se tiene en cuenta sus necesidades específicas en función de su discapacidad, por ejemplo, las mujeres con discapacidad auditiva usuarias de lengua de señas, cuando van solas a una consulta médica, perciben que están perdiendo el tiempo, por cuanto el médico no entiende lo que intentan expresar, lo que sienten, y ellas tampoco comprenden lo que el médico pretende comunicarles. En segundo lugar, pero relacionado con lo anterior, las mujeres con discapacidad, independientemente de su discapacidad, se perciben "invisibilizadas" por los profesionales de la salud, ya que cuando asisten a consulta, acompañadas, los profesionales se dirigen a la persona que está con ellas para preguntar o formular algún tipo de recomendación en torno al tratamiento, como si las mujeres fuesen incapaces de dar cuenta de su condición de salud, de lo que piensan y sienten.

Los hallazgos de este estudio y las conclusiones a las que se llegaron, interpelan a los profesionales y a las organizaciones que trabajan en la atención psicosocial a mujeres con discapacidad respecto a la necesidad de responder de manera real y efectiva a sus necesidades, y a los investigadores, para continuar generando conocimiento basado en la evidencia, que permita avanzar en la construcción de políticas encaminadas a garantizar una verdadera inclusión social de este colectivo. Particularmente, se espera desarrollar una segunda fase de este estudio en la que se incluyan mujeres con otras discapacidades y también, niñas con discapacidad. Adicionalmente, se espera, en consonancia con el modelo social de la discapacidad, formular propuestas de investigación en esta temática con la participación activa de mujeres con discapacidad, desde la propia gestación de la idea de investigación. 


\section{REFERENCIAS}

Arcos, E., Figueroa, V., Miranda, C., y Ramos, C. (2007). Estado del Arte y Fundamentos para la Construcción de Indicadores de Género en Educación. Estudios Pedagógicos, XXXIII-2,121-130. Recuperado el 25 de abril de 2009 de http://www.scielo.cl/pdf/estped/v33n2/arto7.pdf

Banks, M. (2003). Preface. In: M.E. Banks and E. Kaschk (Eds.).Women with Visible and Invisible Disabilities: Multiple Intersections, Multiple Issues, Multiple Therapies (pp. xxi-xxxix). New York: The Haworth Press, Inc.

Bericat, E. (1998). La Integración de los Métodos Cualitativo y Cuantitativo en la Investigación Social. Barcelona: Ariel.

Cisternas, M. S. (2013). Salud global, género y derechos humanos. Revista enfoques, 11(18), 153-186. Recuperado el 30 de julio de 2014 de http:// www.ucentral.cl/prontus_ucentral2012/site/artic/20130731/asocfile/20130731181204/enfoques18mariasoledadcisternas.pdf

Confederación Española de Personas con Discapacidad Física y Orgánica.-COCEMFE-(2003). Libro verde: Mujer y Discapacidad: Propuesta para una década. Madrid: Autor.

Cruz, M.P. (2004). Mujeres con Discapacidad y su Derecho a la Sexualidad. Política y Cultura, 22, 147-160. Recuperado el 25 de abril de 2009 de http://148.206.107.15/biblioteca_digital/estadistica.php?id_hos$\mathrm{t}=6 \&$ tipo $=$ ARTICULO\&id=2387\&archivo $=8-153-2387 \mathrm{tds}$.pdf\&titulo=Mujeres $\% 20$ con $\% 2$ odiscapacidad $\% 20 y \% 20$ su $\% 2$ oderecho $\% 20 a \% 20$ la\%2osexualidad.

Feldman, S.\& Tegart, G.(2003). Conceptions of Illness and Disability of Middle-Aged African-American Women with Arthritis. In: M.E. Banksand E. Kaschk (Eds.). Women with Visible and Invisible Disabilities: Multiple Intersections, Multiple Issues, Multiple Therapies. (pp. 127143). New York: The Haworth Press, Inc.

Giménez, D. \& Ramos, M. (2003). La discriminación de las mujeres discapacitadas en España. Revista del Ministerio de Trabajo e Inmigración, $45,61-76$.

Glasser, B. G., \& Strauss, A. (1967). The discovery of grounded theory: Strategies for qualitativeresearch. Chicago: Aldine.

Gordon, P., Feldman, D., \&Crose, R. (1998). The Meaning of Disability: How Women with Chronic Illness View Their Experiences. The Journal of Rehabilitation, 64, 5-11.

IMSERSO, Universidad Autónoma de Barcelona - Asociació Dones No Estàndars. (2002). Indicadores de exclusión social de mujer y discapacidad. Barcelona. Recuperado el 7 de Marzo del 2009 de http://www. asoc-ies.org/docs/indiexso_muj_dis.pdf

Ley 1090. (2006). Código Deontológico y Bioético. Congreso de la República de Colombia.
López, M. (2008). Mujeres con discapacidad. Mitos y realidades en las relaciones de pareja y en la maternidad. Madrid: Narcea.

Lucas-Carrasco, R. (1998). La versión española del WHOQOL. Madrid: Ed. Ergón.

Lucas-Carrasco, R. (2007). Internacional perspectiva on quality of life in older adults. The WHOQOL-OLD project. Vertex, 18, 130-137.

Lucas-Carrasco, R.; Eser, E.; Hao, Y.; McPherson, K. M.; Green, A.; Kullmann, L. (2011). Research in Developmental Disabilities: A Multidisciplinary Journal, 32, 1212-1225.

Organización de las Naciones Unidas (1979). Convención sobre la eliminación de todas las formas de discriminación contra la mujer (CEDAW). División para el Avance de la Mujer. Recuperado el 30 de septiembre de 2009 de http://www.un.org/womenwatch/daw/cedaw/text/sconvention.htm

Organización de las Naciones Unidas (1993). Normas Uniformes Sobre la Igualdad de Oportunidades para las Personas con Discapacidad. Recuperado el 21 de Octubre de 2009 de: http://www.un.org/esa/socdev/enable/ dissreso.htm

Organización de las Naciones Unidas (2006). Convención Internacional sobre los Derechos de las Personas con Discapacidad y Protocolo Facultativo. Recuperado el 9 de noviembre de 2009 de:http://www.un.org/disabilities/documents/convention/convoptprot-s.pdf

Organización Mundial de la Salud (2001). Clasificación Internacional del Funcionamiento, de la Discapacidad y de la Salud «CIF». Ginebra: Autor.

Power, M.J., Bullinger, M., Harper, A. \& The WHOQOL Group. (1999). The World Health Organization WHOQOL-100: Tests of the Universality of Quality of Life in Fifteen Different Cultural Groups World-wide. Health Psychology, 18, 495-505.

Power, M.J., Green, A.M \& WHOQOL-Dis Group. (2010a). The Attitudes to Disability Scale (ADS): development and psychometric properties. Journal of Intellectual Disability Research, 54, 860-874.

Power, M.J., Green, A.M.\& WHOQOL-Dis Group (2010b). Development of the WHOQOL disabilites module. Quality of Life Research, 19, 571-84.

Power, M. Quinn, K. Schmidt, S. y the WHOQOL-OLD Group (2005). Development of the WHOQOL-OLD module. Quality of Life Research. $14,2197-2214$.

Quinn, G. \& Degener, T. (2002). Uso actual y Posibilidades Futuras de los Instrumentos de Derechos Humanos de las Naciones Unidas en el Contexto de la Discapacidad. Recuperado el 11 de Abril de 2009 de http://www2.ohchr. org/spanish/about/publications/docs/disability.pdf

Ramiro, P. (2004, mayo). Género y discapacidad. Seminario Violencia de Género y Discapacidades. Victoria, España. Recuperado el día 7 de 
mayo de 2010 de http://www.ararteko.net/RecursosWeb/DOCUMENTOS/1/0_401_1.pdf

Samaniego, P. (2006). Aproximación a la realidad de las personas con discapacidad en Latinoamérica. Recuperado el día 30 de marzo de 2010 en la página http://www.discapnet.es/Castellano/comunidad/websocial/Recursos/Documentos/Tecnica/Documents/79216aa9238145598a639eo518e5d808Aproximacionalarealidad.pdf

Schalock, R.L. (2003). El paradigma emergente de la discapacidad y sus retos en este campo. En M.A. Verdugo, y F.B. Jordan de Urríes Vega (Eds.). Investigación, innovación y cambio. V Jornadas Científicas de Investigación sobre personas con discapacidad. (pp.193-233).Salamanca: Amarú.

Skevington, S.M., Lotfy, M., O'Connell, K.A. \& WHOQOL-Group (2004). The World Health Organization's WHOQOL-BREF quality of life assessment: psychometric properties and results of the international field trial. A report from the WHOQOL group. Quality of Life Researcho, 13, 299-310.

Soler, A., Teixeira, T.C. y Jaime, V. (2008, marzo). Discapacidad y dependencia: una perspectiva de género. XI Jornadas de Economía Crítica. Bilbao, España.

Strauss, A., \& Corbin, J. (1990). Basics of qualitative research: Grounded theory procedures andtechniques. Newbury Park, CA: Sage.

Strauss, A., \& Corbin, J. (1998). Basics of qualitative research: Techniques and procedures for developing grounded theory. $\left(2^{\text {nd }}\right.$. ed.).Newbury Park, CA: Sage.

Teddlie, Ch. \& Tashakkori, A. (2006). A General Typology of Research Designs Featuring Mixed Methods, Research in the Schools, 13, 12-28.

The WHOQOL Group. (1998). The World Health Organization Quality of Life Assessment (WHOQOL): Development and general psychometric properties. Social Science \& Medicine, 46, 1569-1585.

UN (2008). The New Image- Medium for Promoting Equalisation of Opportunities and Rights. Documento Vox Nostra, Itegrator, 17, 1-24.
Verdugo, M.A. (2003). La concepción de discapacidad en los modelos sociales. En: M.A. Verdugo y F.B.Jordan de Urríes Vega (eds.). Investigación, innovación y cambio. V Jornadas Científicas de Investigación sobre personas con discapacidad (pp.235-247) .Salamanca: Amarú.

Victoria, J. (2013). Hacia un modelo de atención a la discapacidad basado en los derechos humanos. Boletín Mexicano de derecho comparado, 138, 1093-1109. Recuperado el 30 de julio de 2014 de http://biblio.juridicas. unam.mx/revista/pdf/DerechoComparado/138/art/art8.pdf

WHO-Division of Mental Health and Prevention of Substance Abuse (1998). WHOQOL User Manual, WHO, Geneva.

WHOQOL Group. (1995). The World Health Organization Quality of Life assessment (WHOQOL); position paper from the World Health Organization. Social Science \& Medicine, 41, 1403-1409.

WHOQOL Group. (1998). Development of the World Health Organization WHOQOL-BREF Quality of Life Assessment. Psychological medicine, $28,551-558$.

Williams, M. \& Upadhyay, W. (2003). To Be or Not to Be Disabled. In M.E. Banksand E. Kaschk (Eds.). Women with Visible and Invisible Disabilities: Multiple Intersections, Multiple Issues, Multiple Therapies (pp. 145154). New York: The Haworth Press, Inc

Agradecimientos:

A: AECID, Universidad de La Sabana y Universidad de Barcelona, por haber financiado el proyecto «CREENCIAS Y PRACTICAS DE MUJERES CON DISCAPACIDAD DESDE LA PERSPECTIVA DE DERECHOS»; Organizaciones de y para personas con discapacidad de las ciudades de Bogotá, Medellín, Cali y Barranquilla; y especialmente, a las mujeres con discapacidad, que participaron en el Proyecto.
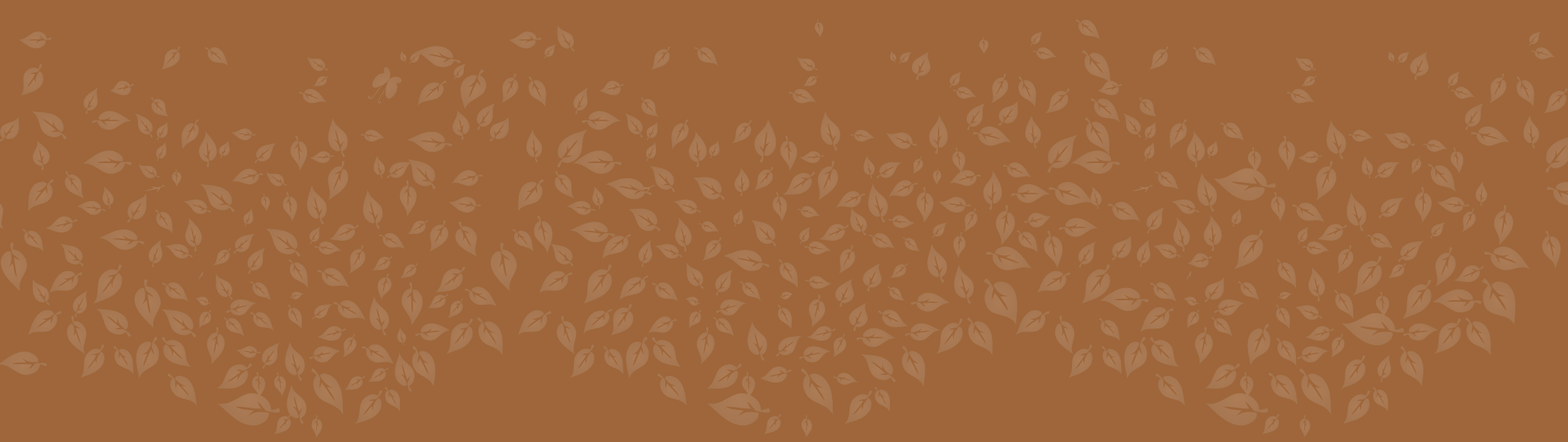

REV. COL. REH 2014 || Volumen 13 || Páginas 4 - 13 || 